\title{
ARTICLE Disrupting reconsolidation: memory erasure or blunting of emotional/motivational value?
}

\author{
Elizabeth S. Cogan ${ }^{1}$, Mark A. Shapses ${ }^{1}$, Terry E. Robinson (iD) ${ }^{1}$ and Natalie C. Tronson (iD)
}

\begin{abstract}
When memories are retrieved they become labile, and subject to alteration by a process known as reconsolidation. Disruption of memory reconsolidation decreases the performance of learned responses, which is often attributed to erasure of the memory; in the case of Pavlovian learning, to a loss of the association between a conditioned stimulus (CS) and unconditioned stimulus (US). However, an alternative interpretation is that disrupting reconsolidation does not erase memories, but blunts their emotional/ motivational impact. It is difficult to parse the predictive vs. emotional/motivational value of CSs in non-human animals, but studies on variation in the form of conditioned responses (CRs) in a Pavlovian conditioned approach task suggest a way to do this. In this task a lever-CS paired with a food reward (US) acquires predictive value in all rats, but is attributed with emotional/motivational value to a greater extent in some rats (sign-trackers) than others (goal-trackers). We report that the post-retrieval administration of propranolol selectively attenuates a sign-tracking $C R$, and the associated neural activation of brain "motive circuits", while having no effect on conditioned orienting behavior in sign-trackers, or on goal-tracking CRs evoked by either a lever-CS or a tone-CS. We conclude that the disruption of reconsolidation by post-retrieval propranolol degrades the emotional/motivational impact of the CS, required for sign-tracking, but leaves the CS-US association intact. The possibility that post-retrieval interventions can reduce the emotional/motivational aspects of memories, without actually erasing them, has important implications for treating maladaptive memories that contribute to some psychiatric disorders.
\end{abstract}

Neuropsychopharmacology (2019) 44:399-407; https://doi.org/10.1038/s41386-018-0082-0

\section{INTRODUCTION}

When memories are retrieved they become subject to modification by a process known as reconsolidation. During the reconsolidation "window" memories can be altered-updated, enhanced, or disrupted-by manipulations of molecular mechanisms of plasticity and memory stability [1, 2]. There has been considerable interest, therefore, in the possibility of disrupting the reconsolidation of maladaptive memories to treat disorders such as post-traumatic stress disorder and addiction [3-5]. However, it is still unclear exactly what aspects of memory are affected when reconsolidation is disrupted.

In Pavlovian learning, commonly used to study reconsolidation, the dominant view is that the association between a conditioned stimulus (CS) and unconditioned stimulus (US) is strengthened, modified, or erased. Thus, in the case of erasure, the ability of the CS to predict the US is lost $[3,6,7]$. This interpretation comes largely from Pavlovian fear conditioning studies in animals, in which the disruption of reconsolidation is manifest by a decrease in the ability of a CS to evoke a simple conditioned response (CR), such as freezing $[1,2,8,9]$. However, studies of fear conditioning in humans suggest that disruption of reconsolidation may not actually erase memory. For example, the post-retrieval administration of the $\beta$-adrenergic antagonist propranolol reduces the expression of a conditioned fear potentiated startle response in humans, but the participants' declarative expectation that shock follows the CS remains intact $[10,11]$.
This dissociation between an intact declarative memory of the CS-US association, but a decrease in a CS-evoked CR, raises the possibility that disrupting reconsolidation may not erase memories, but rather, may degrade the emotional/motivational impact of the CS [12]. Thus far, this idea has not been investigated in the context of appetitive learning, in any species. It is, of course, difficult to parse the predictive vs. emotional/motivational components of a memory in animals, but studies using an appetitive Pavlovian conditioned approach task provide a way to do this $[13,14]$. When a lever-CS is paired with a food US, the lever-CS itself becomes attractive in some rats, and they begin to approach it (sign-trackers, "STs" [15]), whereas in other rats the lever-CS evokes a CR directed toward the location of impending food delivery (goal-trackers, "GTs" [16]). In addition, the lever-CS is a more effective conditioned reinforcer [17], more effective in reinstating reward-seeking [18, 19], and its presentation more strongly engages brain systems that confer emotional/motivational value to CSs, in STs than GTs [20-22]. Thus, the lever-CS is equally predictive in STs and GTs, it evokes a CR in both, but it additionally acquires emotional/motivational value, and the ability to act as an attractive and desired incentive stimulus, to a greater degree in STs [14].

There are individual differences in susceptibility to disruption of reconsolidation $[6,23,24]$, and we asked whether these might be due, at least in part, to variation in the propensity to attribute motivational value to a CS. We hypothesized that if disrupting reconsolidation with propranolol erases the CS-US association,

\footnotetext{
${ }^{1}$ Biopsychology Program, Department of Psychology, University of Michigan, 530 Church Street, Ann Arbor, MI 48109, USA
}

Correspondence: Natalie C. Tronson (ntronson@umich.edu)

Received: 18 December 2017 Revised: 23 March 2018 Accepted: 24 April 2018

Published online: 3 May 2018 
then the ability of a lever-CS to evoke both ST and GT CRs should be disrupted. In contrast, if post-retrieval propranolol blunts the emotional/motivational value of a lever-CS, but leaves the CS-US association intact, then it should selectively disrupt the performance of a ST CR, and the ability of the CS to activate brain regions that confer emotional/motivational value to CSs in STs.

\section{METHODS}

See SI for detailed methods.

Male Sprague-Dawley rats were used in all experiments.

Exp. 1. The effect of post-retrieval propranolol on ST and GT behavior

Rats were trained for 5 days ( 25 trials/day) on a well-characterized Pavlovian conditioned approach task, in which an illuminated lever (the CS) was presented for $8 \mathrm{~s}$, and immediately following its retraction a banana-flavored food pellet (the US) was delivered into an adjacent food cup, on a VT 90 (30-150 s) schedule [25]. Based on their performance rats were classed as STs, who preferentially approached the lever-CS during the $8 \mathrm{~s}$ CS period, or GTs, who instead approached the food cup, using an index described by Meyer et al. [25]. The sessions on days 6 and 7 served as retrieval sessions and were identical to initial Pavlovian training, with the exception that immediately after the end of the session, and before being returned to their home cages, animals received an intraperitoneal (IP) injection of either the $\beta$-adrenergic antagonist, propranolol, or saline. The effect of propranolol was then tested on one additional test day (day 8), the same as during training.

Exp. 2. Non-retrieval control

To determine if memory retrieval was required for propranolol to disrupt reconsolidation a non-retrieval control procedure was used. For this experiment rats were not placed into the conditioning chambers on days 6 and 7, but remained in their home cages, where they received an IP injection of either propranolol or saline at the same time of day as their previous conditioning sessions, and the effects of propranolol assessed on a final session on day 8 .

Exp. 3. The effect of post-retrieval nadolol on ST and GT behavior Nadolol has the same peripheral effects as propranolol but does not cross the blood-brain barrier, and was thus used to determine if the effects of propranolol on reconsolidation were due to peripheral effects (e.g., sympathetic arousal) or due to effects on $\beta$-adrenergic antagonism in the brain. Procedures were identical to those described for Exp. 1, with the exception that animals received nadolol or saline.

Exp. 4. Video analysis of behavior in STs

This experiment was conducted as Exp. 1, but only STs were used, and in this experiment behavior on day 8 was additionally scored by inspection of videos. Three behaviors were quantified: (1) an orienting response; (2) an approach response; and (3) a lever deflection (computer-scored).

Exp. 5. The effect of post-retrieval propranolol on GT behavior evoked by a tone-CS

All Pavlovian training sessions (days 1-5), retrieval and postsession injections (days 6-7), and test session (day 8) were identical to those in Exp. 1, with the exception that a tone-CS (2.9 $\mathrm{kHz}, 70 \mathrm{dbs}$ ), instead of a lever, was presented for $8 \mathrm{~s}$ prior to delivery of the food US.

Exp. 6. The effect of post-retrieval propranolol on GT behavior evoked by a tone-CS in STs and GTs

Pavlovian training sessions (days 1-5) were identical to those described above using a lever-CS to classify rats as STs or GTs.
Following this, all rats were additionally trained for 5 more days to associate a tone-CS $(2.9 \mathrm{kHz}, 70 \mathrm{dbs})$ with the food US (days 6-10), followed by retrieval and post-session injections (days 11-12), and test session (day 13), as in Exp. 5.

Exp. 7. c-Fos evoked by lever-CS in GTs, STs, and STs treated with propranolol

Rats were assigned to "lever paired" ( $n=28$ ) or "lever unpaired" ( $n$ $=7$ ) groups, and then trained as described in Exp. 1, except rats in the unpaired group, for whom the 25 lever-CS presentations and 25 US presentations occurred independently (on separate VT 90-s schedules). Sessions 6-8 served as retrieval sessions, and STs and unpaired animals received a post-session injection of either propranolol or saline. All GTs received saline. After habituation to the context alone for 4 days [21, 22], on the test day (day 13) animals were placed into the test chamber for $5 \mathrm{~min}$ and the CS was presented 10 times ( $4 \mathrm{~s}$ each), one per minute (no US), and then returned to their home cages for $60 \mathrm{~min}$ before their brains were obtained and processed for c-Fos + .

Exp. 8. The ability of a tone-CS to evoke c-Fos

This experiment was conducted as per Exp. 6, with a separate cohort of animals. A tone-CS was Paired or Unpaired with presentation of the food US for 8 days, followed by 4 context extinction sessions. On the test day (day 13) all animals received 10 tone-CS (4 s each) presentations, once per minute (no US), and 60 min later brains were obtained and processed to examine c-Fos + cells.

\section{RESULTS}

See SI for statistics.

Exp. 1. Disruption of reconsolidation by propranolol selectively decreases ST behavior

Across training days $1-5$, animals classified as STs engaged the lever-CS significantly more than GTs (Fig. 1c-e), whereas animals classified as GTs interacted with the food cup significantly more than the STs (Fig. 1f-h; see SI).

When given after memory retrieval on days 6 and 7, propranolol significantly reduced lever-directed behavior in STs (Fig. 1C-e; treatment $\times$ session; contacts: $F_{2,102}=9.26, P<0.001$; probability: $F_{2,65}=9.07, P<0.001$; latency: $\left.F_{2,68}=10.50, P<0.001\right)$. In contrast, post-retrieval propranolol had no significant effect on GT behavior in GTs (Fig. 1f-h; contacts: $F_{2,102}=0.33, P=0.72$; probability: $F_{2,72}$ $=0.17, P=0.85$; latency: $\left.F_{2,69}=0.62, P=0.54\right)$. Thus, post-retrieval propranolol selectively decreased the performance of a ST CR in STs while having no effect on the performance of a GT CR in GTs.

\section{Exp. 2. Disruption of reconsolidation by propranolol requires} memory retrieval

The acquisition of ST and GT behavior was similar to Exp. 1 (Fig. $2 a-c)$. The administration of propranolol without memory retrieval had no effect on lever-directed behavior in STs on day 8 (Fig. 2b, c; Fig. S1 A-D; no treatment $\times$ session interaction; contacts: $F_{1,30}=0.148, \quad P=0.703 ; \quad$ probability: $F_{1,30}=0.222, \quad P=0.641$; latency: $F_{1,30}=0.003, P=0.959$ ), or food cup-directed behavior in GTs (Fig. 2b, c; Fig. S1 A-D; no treatment $\times$ session interaction; contacts: $\quad F_{1,30}=1.831, \quad P=0.186 ;$ probability: $F_{1,30}=0.003$, $P=0.957$; latency: $F_{1,30}=0.731, P=0.399$ ). Therefore, memory retrieval (achieved by placement into the test chambers prior to propranolol administration on days 6 and 7 in Exp. 1) was necessary for propranolol to disrupt ST behavior.

\section{Exp. 3. Nadolol does not disrupt reconsolidation}

The acquisition of ST and GT behavior was similar to Exp. 1 (Fig. 2d-f). In contrast to propranolol (Exp. 1), nadolol, which does not cross the blood-brain barrier, had no effect on lever-directed 


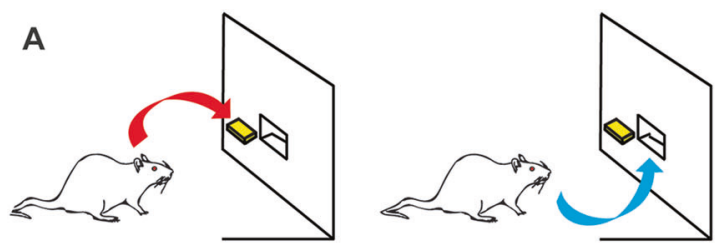

B Experimental Timeline
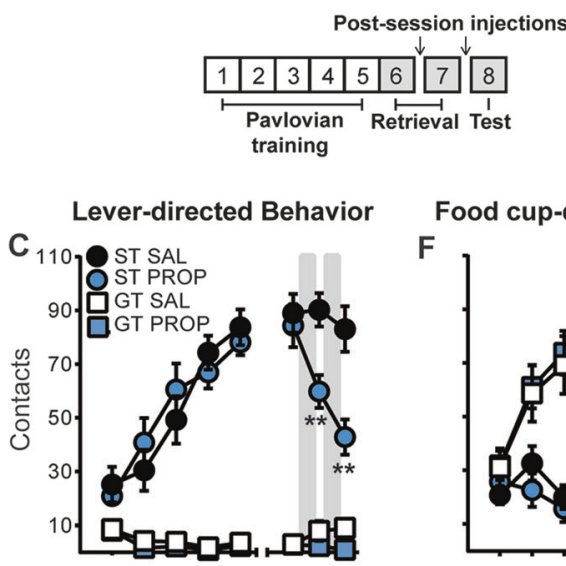

Food cup-directed Behavior

$\mathbf{F}$
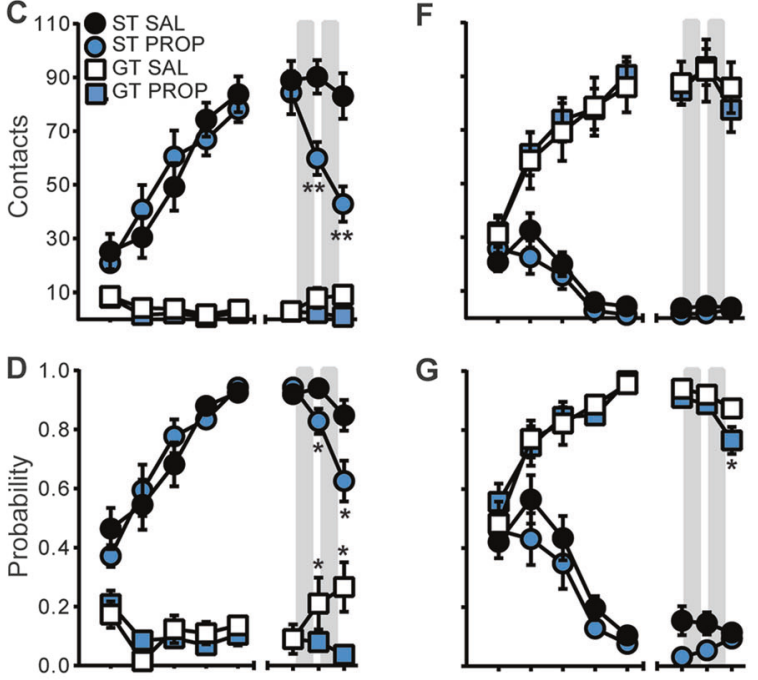

G
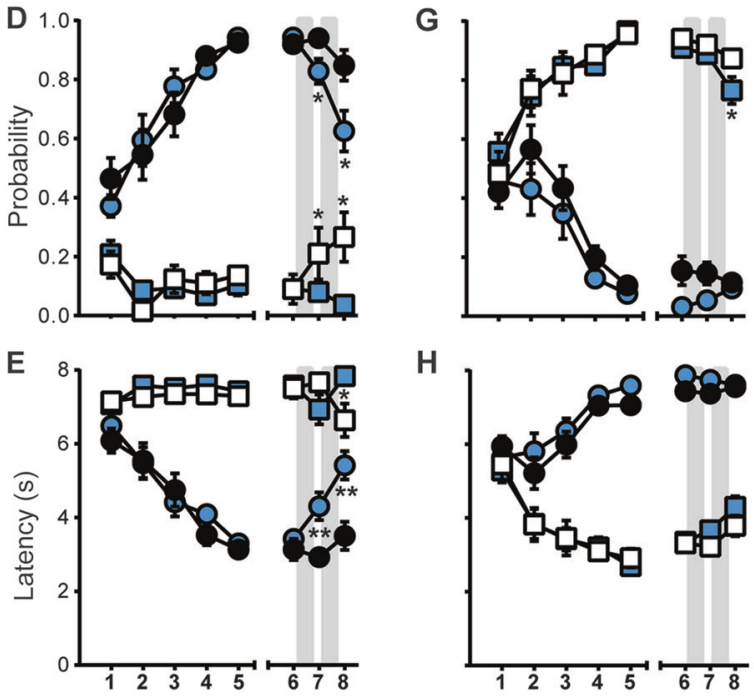

$\mathrm{H}$

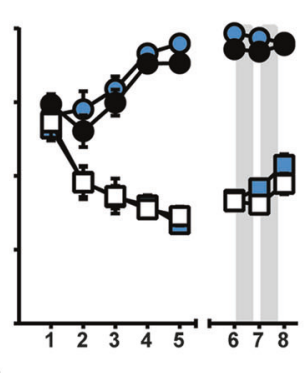

Fig. 1 Disruption of reconsolidation by propranolol selectively decreases STs. a Illustration of lever-directed behavior (sign-tracking; left) and food cup-directed behavior (goal-tracking; right) during the presentation of a lever-CS. b Experimental timeline indicating that the injections were administered after retrieval sessions on days 6 and 7. c-e Lever-directed behavior, and $\mathbf{f}-\mathbf{h}$ food cup-directed behavior, in all groups across sessions 1-8 (sessions 1-5, training; sessions 6-7, retrieval; and session 8, test). Gray bars represent the administration of propranolol (PROP) or saline (SAL) injections after sessions 6 and 7. Data represent mean \pm SEM. ${ }^{*} P<0.01,{ }^{* *} P<0.001$ (relative to session 6)

behavior in STs (Fig. 2e, f; Fig S1 E-H; no treatment $\times$ session interaction; contacts: $F_{2,93}=0.031, P=0.969$; probability: $F_{2,61}=$ $0.605, P=0.550$; latency: $F_{2,67}=0.016, P=0.984$ ) nor food cupdirected behavior in GTs (Fig. 2e, f; Fig S1 E-H; no treatment $\times$ session interaction; contacts: $F_{2,94}=0.777, P=0.463$; probability: $F_{2,92}=0.193, P=0.825$; latency: $F_{2,80}=0.116, P=0.89$ ). Thus, the disruption of sign-tracking behavior by propranolol requires central antagonism of $\beta$-adrenergic receptors.

\section{A Experimental Timeline}

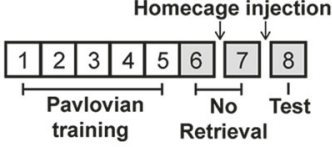

Lever-directed Behavior Food cup-directed Behavior

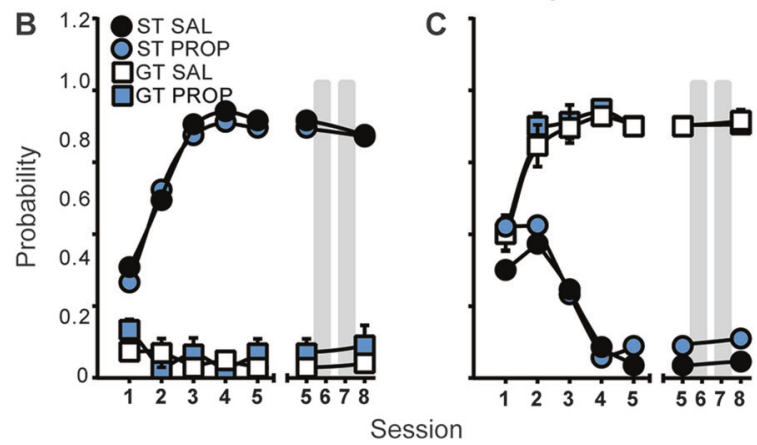

D Experimental Timeline

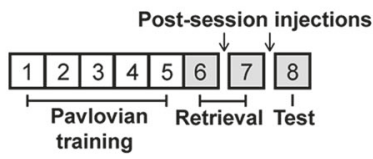

Lever-directed Behavior

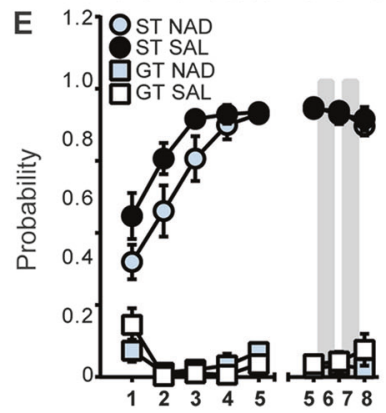

Food cup-directed Behavior

$\mathrm{F}$

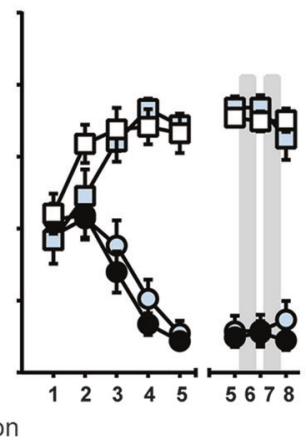

Fig. 2 Disruption of reconsolidation by PROP requires memory retrieval and action in central nervous system. a Experimental timeline indicating that injections were administered in the home cage, with no retrieval sessions. b Probability of making a lever contact. c Probability of food cup-directed behavior across sessions $1-8$. Note on the right, behavior on the test day (day 8 ) is compared to that on the final training day (day 5) because no behavioral testing was conducted on days 6 and 7. d Experimental timeline. e Probability of making a lever contact. $\mathbf{f}$ Probability of food cupdirected behavior across sessions $1-8$, as described in Fig. 1. NAD nadolol. Data represent mean \pm SEM

Exp. 4. Propranolol disrupts reconsolidation of ST, but not conditioned orienting in STs

To further determine whether the CS-US association remained intact in STs despite reduction of the ST CR, we examined conditioned orienting behavior. Although only STs approach into close proximity to the CS, and engage with it, both STs and GTs develop a conditioned orienting response to a CS that predicts either food or drug, and this CR does not appear to reflect the motivational value of the CS $[22,26]$.

Between acquisition days 1 and 5, STs that received paired presentations of the CS and US showed an increase in the number of lever contacts (deflections; Fig. 3a, left) and a decrease in the latency to contact the lever (Fig. 3b, left), as measured automatically, and there were no differences in the groups to 
Lever-directed Behavior

A

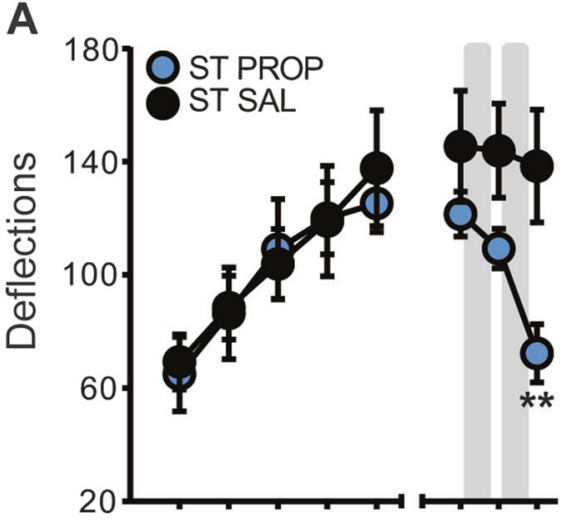

B

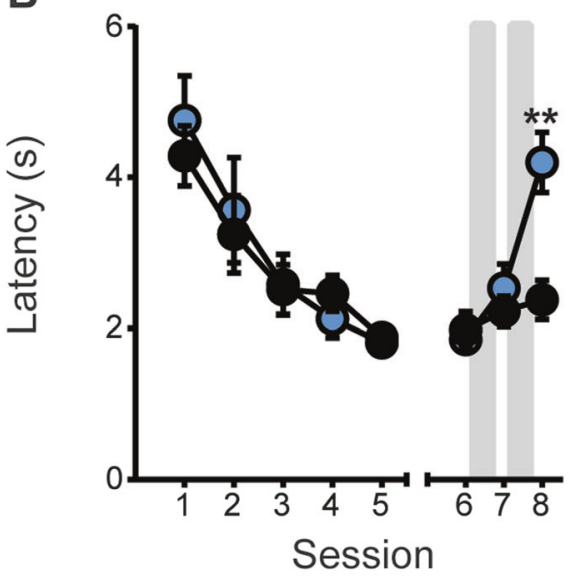

Final Session Trials 1-25

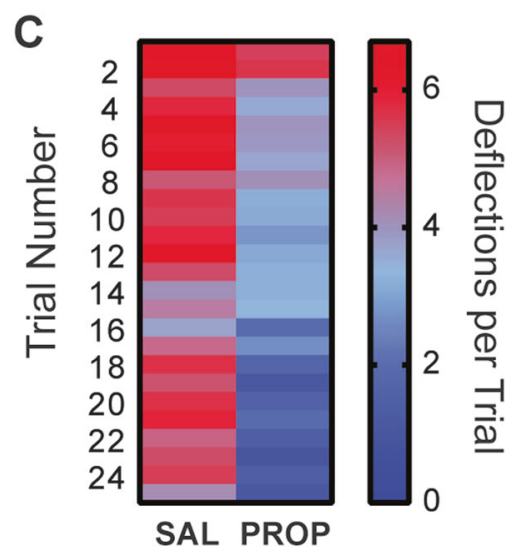

D

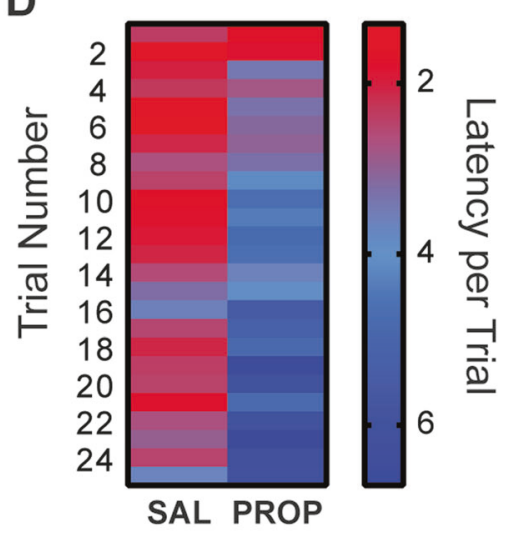

$\mathbf{E}$

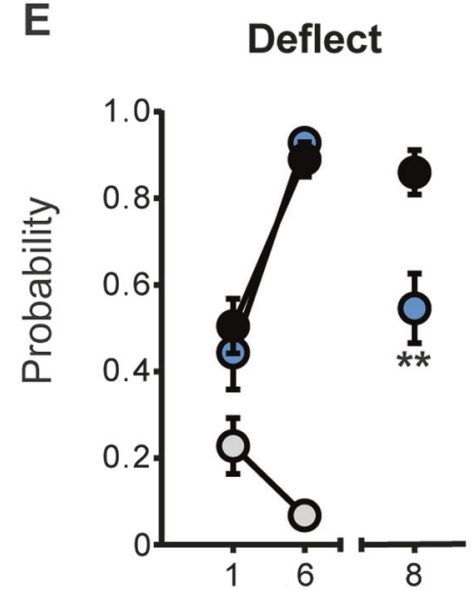

$\mathbf{F}$

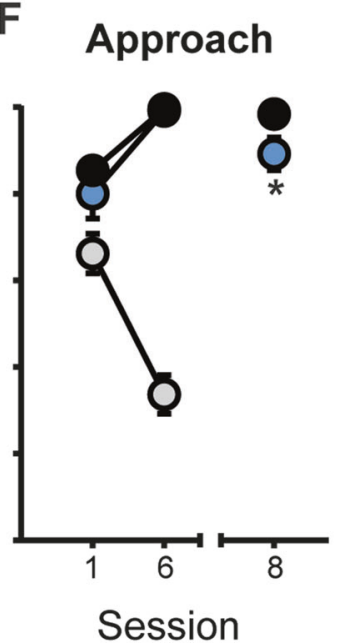

G Orient

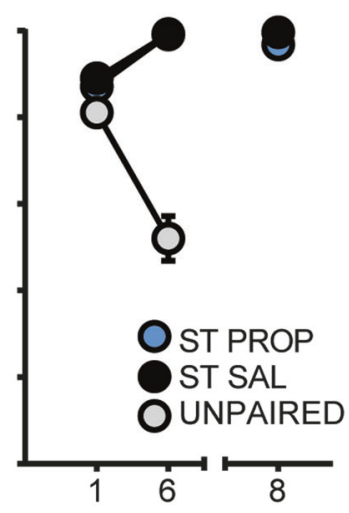

Fig. 3 Propranolol disrupts reconsolidation of ST, but not conditioned orienting. Lever-directed behavior (a contacts and $\mathbf{b}$ latency) across sessions 1-8, as described in Fig. 1. c, $\mathbf{d}$ Heat map representations of the number of lever contacts and latency to contact the lever during each of the 25 trials on test session 8. e, $\mathbf{f}$ The probability of making a lever deflection (e), an approach response (f), or an orienting response (g) on the first (day 1), last (day 6) training day, and on final test day 8, in STs after treatment with propranolol (PROP) or saline (SAL), and in unpaired animals. ${ }^{*} P<0.05,{ }^{*} P<0.001$ (relative to session 6 )

receive propranolol or saline. Figure $3 \mathrm{e}-\mathrm{g}$ shows further analyses of the animals' behavior, incorporating data obtained from videos, and comparing paired and unpaired groups. Figure 3e shows that the probability of contacting the lever (measured automatically as a lever deflection), making a video scored approach response (did not require lever deflection), and video scored orienting response increased in paired, but not in unpaired groups, over the 6 days of training. The high level of orienting to the lever on the first day of 
A

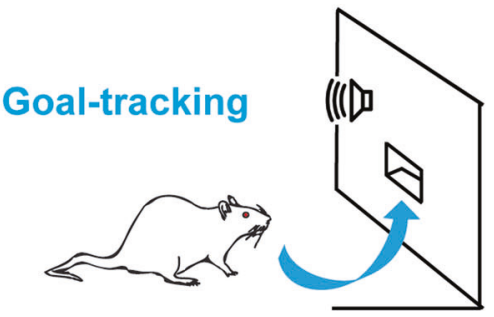

B Experimental Timeline

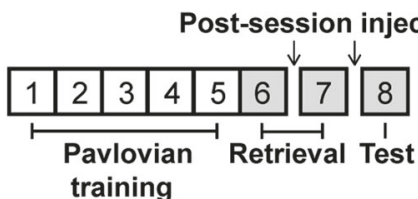

Food cup-directed Behavior

C
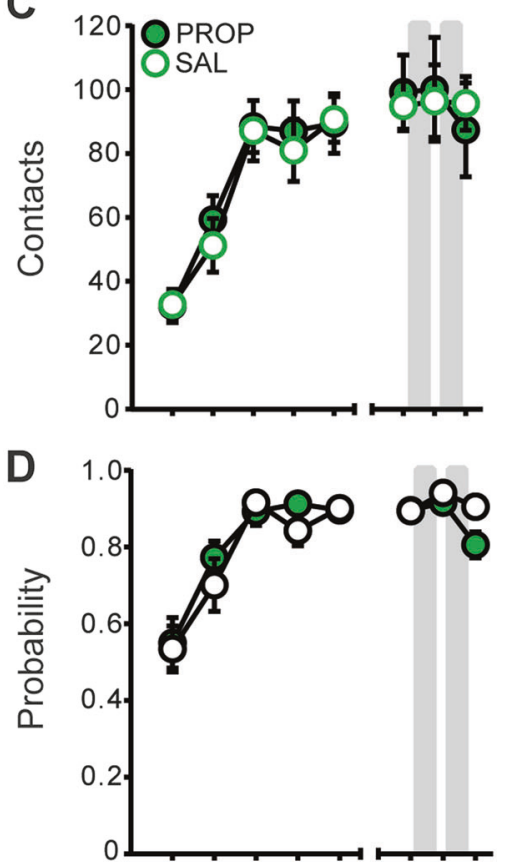

$\mathbf{E}$

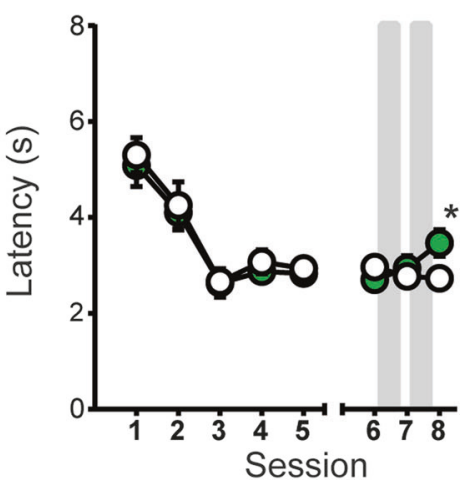

Fig. 4 PROP does not disrupt GT evoked by a tone-CS. a Illustration of a goal-tracking CR to the presentation of a tone-CS. b Experimental timeline. c-e Goal-tracking behavior (c number of food cup entries; d probability; e latency) across sessions $1-8$. Data represent mean \pm SEM. ${ }^{*} P<0.05$ training was due to stimulus novelty. Nevertheless, the comparison between the unpaired and paired groups over days of training indicates that by day 6 orienting behavior represents an acquired CR (Fig. 3g).

As in Exp. 1, post-retrieval propranolol decreased the number of lever contacts and increased the latency to contact the lever in these STs, measured automatically (Fig. 3a, b, right). Post-retrieval propranolol also decreased the probability of a lever deflection (contact) (Fig. 3e) and making a video scored approach response (Fig. 3f) during the CS period. In contrast, propranolol had no effect on conditioned orienting behavior (Fig. 3g). These data suggest that even in STs treated with propranolol the CS-US association remained intact, as it continued to evoke a conditioned orienting response, even as it attenuated ST behavior measured by lever deflections and approach.

The magnitude of decrease in the number of computer-scored lever contacts (deflections; Fig. 3e) was much greater than the decrease in the number of approaches scored from video (which did not require a lever deflection, Fig. 3f). The typical pattern of behavior for STs in the saline-treated group was to initially orient to the lever upon presentation, and then rapidly approach, immediately contact, and vigorously engage it (primarily biting it), as reflected in the computer-generated data (see SI Videos). However, this level of engagement with the lever was diminished in propranolol-treated STs. Upon lever presentation some propranolol-treated STs would orient and stare at it for the remainder of the CS period, without contacting it. Others continued to approach the lever, albeit more slowly, but rather than engaging it vigorously like control rats, they would sniff around the lever and perhaps delicately investigate it with their front paws. Sometimes, but not always, this resulted in a computer-scored lever deflection. Thus, the greater decrease in the probability of approach indicated by automated scoring compared with approach quantified by video analysis was because many propranolol-treated rats continued to approach, but not vigorously engage or deflect, the lever-CS. Importantly, behavior during the CS period remained focused on the lever-CS and the rats did not begin to goal-track (see Fig. S3).

Trial-by-trial analysis of behavior on day 8 also revealed important group differences. On day 8 rats in the saline control group continued to approach the lever rapidly (Fig. 3d), and engaged it vigorously (Fig. 3c), throughout the entire 25 trial session. Propranolol-treated STs continued to approach and engage the lever-CS on the first 1-2 trials of the test session, but this behavior quickly waned. There were no significant differences between the propranolol and saline groups in the number of lever deflections or in the latency to approach the lever, during the first trial on day 8. But in STs treated with propranolol there was a progressive increase in the latency to contact the lever over the course of the session and a decrease in the number of lever deflections (Fig. 3c, d). These data further support the contention that the CS retained predictive value in propranolol-treated STs, as it continued to evoke a ST CR in the first trial. Propranolol-treated STs were thus physically capable of making such responses. Importantly, the decreased lever deflections in propranolol-treated STs cannot be attributed to withinsession extinction as all trials were reinforced and the animals continued to retrieve and eat the food pellets upon lever retraction.

Exp. 5. Post-retrieval propranolol does not disrupt GT evoked by a tone-CS

If a tone-CS is paired with food delivery all rats develop a GT CR [27], even animals that have been screened and classed as STs [28, 29]. Furthermore, a tone-CS is a less-effective conditioned reinforcer than a lever-CS, suggesting it is not attributed with incentive motivational value to the same extent as a lever-CS [2830]. Post-retrieval propranolol had no effect on the number of 


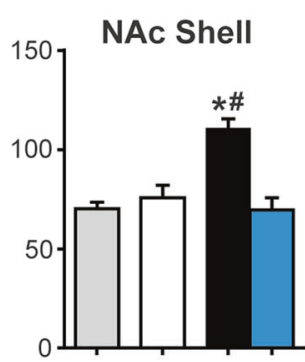

Lateral Septum
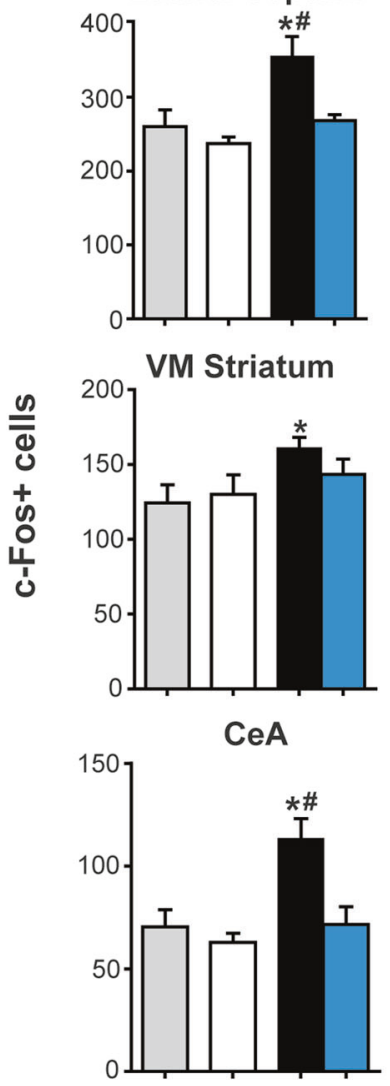

BLA

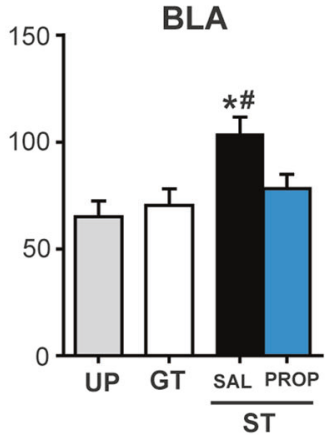

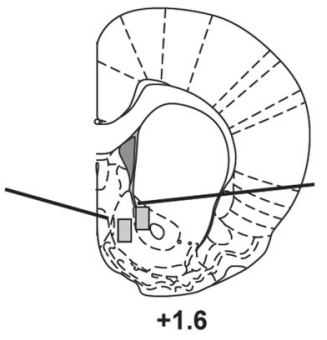

DM Striatum
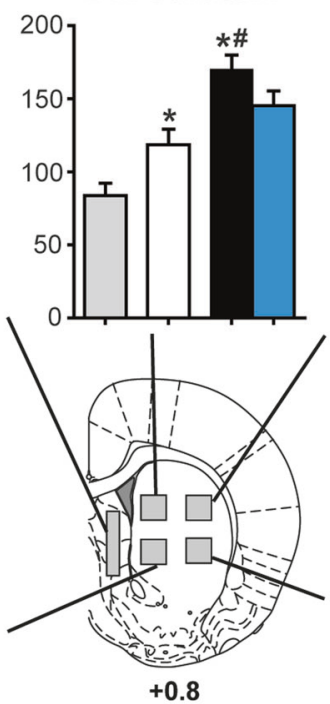

$+0.8$
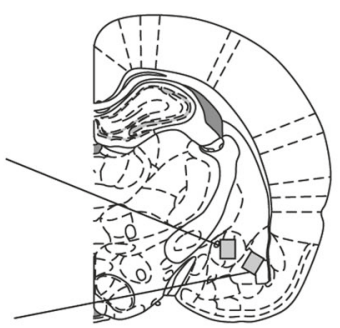

$-2.54$
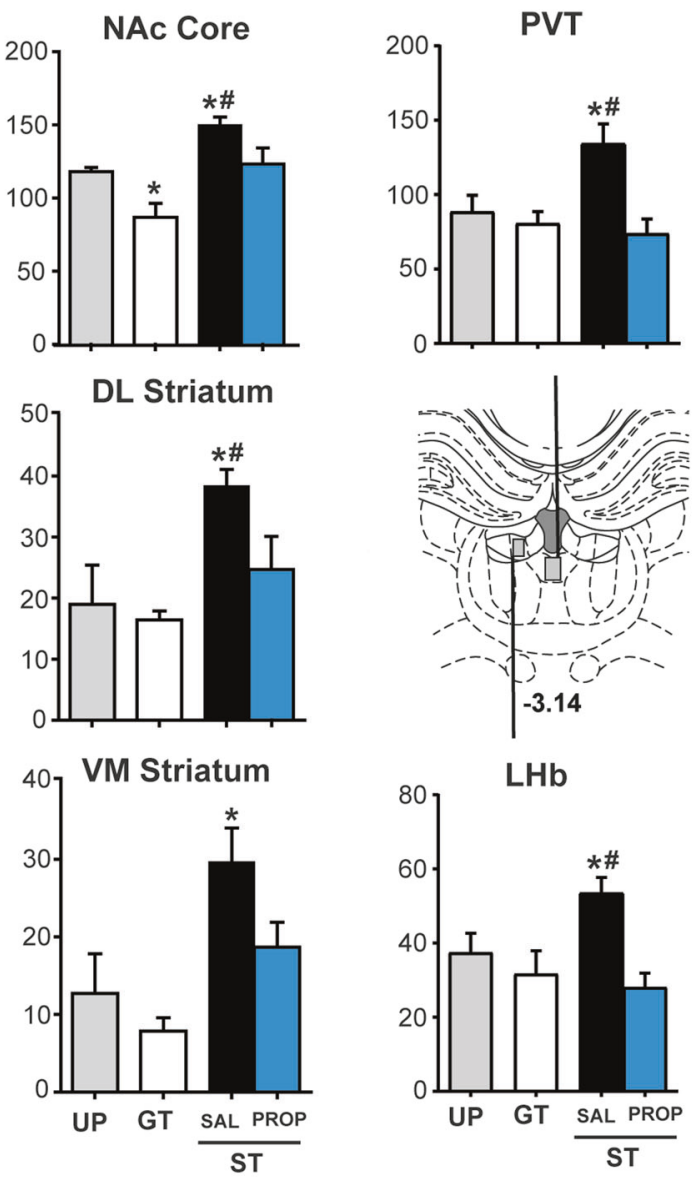

Paraventricular Nucleus of the Thalamus

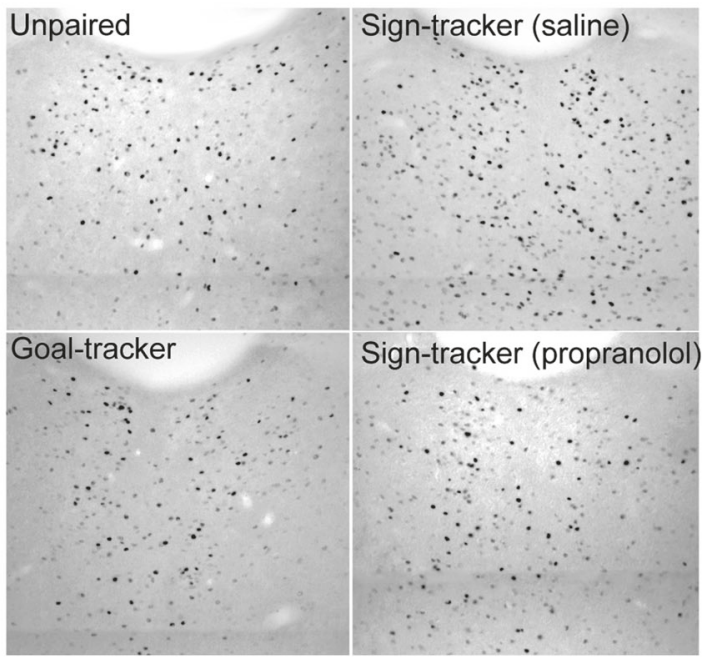

Fig. 5 PROP decreases the ability of a lever-CS to engage brain reward regions in STs. Mean \pm SEM number of c-Fos + cells in the unpaired group (UP), GTs, saline-treated STs (ST-SAL), and propranolol-treated STs (ST-PROP) in all the brain regions analyzed. NAc nucleus accumbens, PVT paraventricular nucleus of the thalamus, DM dorsomedial, DL dorsolateral, VM ventromedial, VL ventrolateral, LHb lateral habenula, CeA central amygdala, BLA basolateral amygdala. A representative image of c-Fos + cells from the PVT in each of the four groups are shown in the lower right portion of the figure. *Significant difference from UP $(P<0.05)$; ${ }^{\#}$ significant difference from ST-PROP $(P<0.05)$

food cup contacts (Fig. 4a-c; no treatment $\times$ session interaction; $F_{2,29}=1.159, P=0.328$ ), probability of a contact (no treatment $\times$ session interaction; $F_{2,42}=2.586, P=0.087$ ), or latency (no effect of treatment; $F_{1,21}=0.607, P=0.445$; no effect of session; $F_{2,42}=1.360, P=0.268$ ) elicited by a tone-CS.
Exp. 6. Post-retrieval propranolol does not disrupt GT evoked by a tone-CS in rats identified as STs and GTs

Post-retrieval propranolol injections did not decrease goaltracking to a tone-CS in rats previously identified as either STs or GTs (no phenotype $\times$ session interaction, contacts: $F_{2,31}=0.310$, 
$P=0.735$; probability: $F_{2,40}=0.183, P=0.834$; latency: $F_{2,40}=$ $1.293, P=0.286$ (see SI and Fig. S2).

Exp. 7. Post-retrieval propranolol decreases the ability of a lever-CS to engage brain reward regions in STs

The acquisition of ST and GT behavior, and the behavioral effect of post-retrieval propranolol, are shown in Fig. S3. During the final test session (day 13) the rats were exposed to 10 presentations of the CS only (no US), and $60 \mathrm{~min}$ later brains were obtained to quantify c-Fos+ cells in selected brain regions (Fig. 5). Consistent with previous findings [21,22], the CS increased the number of cFos + cells in the selected brain regions in STs, relative to unpaired controls and GTs (see SI). However, propranolol-treated STs had significantly fewer c-Fos + cells than saline-treated STs in most brain regions examined.

Exp. 8. A tone-CS did not evoke c-Fos in brain reward regions Presentation of a tone-CS failed to induce c-Fos in all of the brain regions examined in paired rats that had acquired a GT CR, relative to unpaired controls (see SI Fig. S4 and Table S1).

\section{DISCUSSION}

Disruption of memory reconsolidation impairs subsequent performance in a number of learning tasks and species [1, 9, 10, 31-33]. In the case of Pavlovian fear conditioning, this is manifest by a reduction in the ability of a CS to evoke conditioned freezing behavior. This decrement in performance is often interpreted as a loss in the ability of the CS to evoke a representation of the US (the shock), because the memory of that association was erased (e.g., see [34]). But as cautioned by Bouton and Moody [35], "What an animal does in a conditioning experiment is not the same as what it knows. ... Learning is not the same as performance. Motivation is required for the translation" (p. 663-664). An alternative interpretation, supported by studies in humans (e.g., see [10]), is that knowledge of the association between the CS and US remains fully intact, but the CS no longer has sufficient emotional/motivational value to evoke a CR (also see [36]).

Memory erasure or blunting of emotional/motivational impact? It is difficult to parse the emotional/motivational vs. predictive value of an aversive CS in non-human animals. In fact, these elements of memory are confounded in all previous studies of reconsolidation in non-human animals, complicating their interpretation. However, the ST/GT model allows us to investigate the extent to which variation in the emotional/motivational attributes of an appetitive Pavlovian memory contributes to individual differences in susceptibiliy to disruption of reconsolidation $[6,23$, 24]. Variation in the form of the CRs that emerge during Pavlovian conditioned approach training is due, in part, to differences in the propensity of animals to attribute incentive salience to the CS, transforming the CS from a merely predictive stimulus into an emotionally and motivationally significant incentive stimulus [14]. An incentive is quantified by the extent to which a reward cue becomes attractive, desirable, and capable of invigorating seeking behavior [4, 37]. A lever-CS acquires the characteristics of an incentive stimulus to a greater degree in STs than in GTs, and in rats, a tone-CS, which evokes GT, is a less-effective incentive stimulus than a lever-CS [28-30]. Finally, food and drug cues influence neural activity in brain regions that control motivated behavior to a greater extent in STs than in GTs [20-22, 30], and the acquisition and performance of ST, but not GT, is dopaminedependent $[26,38]$.

Given this information, why might post-retrieval propranolol have degraded performance of a ST CR, but not a GT CR? We suggest that propranolol degraded the emotional/motivational value of the lever-CS, while leaving the predictive relationship between the CS and US (the memory) intact. Evidence that propranolol did not erase memory include: (1) GT CRs were not disrupted, whether evoked by the lever-CS in GTs, or a tone-CS in all rats. As discussed above, performance of a ST CR is dependent on the CS being attributed with incentive salience, whereas performance of a GT CR is not. (2) Propranolol did not disrupt performance of a conditioned orienting response to a lever-CS, even in STs, suggesting that even in STs the CS-US association remained intact. (3) STs continued to show anticipatory approach behavior after propranolol treatment, further suggesting the leverCS still had predictive value. However, after propranolol STs approached the lever-CS much less avidly, more slowly, and when they did engage they did so much less vigorously. (4) STs continued to quickly approach and engage the lever-CS on the very first trial of the test session after propranolol treatments (day 8 ), but as the session progressed the number of lever contacts dwindled and the latency to approach increased. The fact that STs continued to approach the lever-CS on the very first trial after propranolol is consistent with the idea that the CS retained predictive, informational value. That this behavior quickly waned suggests that the ability of the CS to generate emotional/ motivational "excitement", to act as a potent incentive stimulus, was much diminished (also see [36]).

The interpretation that post-retrieval propranolol (and not nadolol) disrupted motivational, but not predictive components of memory is also consistent with our observation that propranolol reduced the ability of the lever-CS to engage brain regions that confer incentive value to reward cues in STs. Given the critical role of the motive circuit in the attribution of incentive value, the global decrease c-Fos across these brain regions after disruption of reconsolidation provides strong support for the specific susceptibility of emotional/motivational components of memory. Although the "motive circuit" is usually thought to mediate the expression of motivated behaviors (e.g., [37]), our findings also suggest a potential role for these brain regions in the storage of motivational components of memory.

Our work extends and explains previous findings on differences in susceptibility of STs and GTs to reconsolidation. Blaiss and Janak [39] reported that disruption of reconsolidation failed to decrease GT behavior, and others (e.g., [40]) reported a loss of discrimination between a food paired CS and an unpaired CS, but not a decrease in responding to the predictive CS. Other studies found that the conditioned reinforcing properties of an appetitive CS were attenuated after disruption of reconsolidation, and although this was interpreted as disruption of the associative properties of the CS [31], it is equally consistent with the idea that the motivational, but not associative, properties of the CS were disrupted. Finally, Pasquariello et al. [41] recently reported that post-session propranolol prevented the acquisition of a ST CR, but not a GT CR. Together, these findings demonstrate that emotional/ motivational properties of memory are uniquely susceptible to disruptions of reconsolidation by propranolol, whereas predictive relationships are remarkably stable.

Together with the studies on fear conditioning by Kindt and colleagues (e.g., [10, 11]), these findings suggest that the emotional/motivational properties of both appetitive and aversive Pavlovian memories are susceptible to disruption by propranolol. Whether this is true for other types of memory, and for other disruptors of reconsolidation remains to be tested. It is also notable that in our studies, affective properties of memory were sensitive to disruption despite commonly cited boundary conditions for reconsolidation (e.g., [42]). Yet in our experiments, reactivation consisted of sessions identical to previous training sessions, with no alterations of context or cues providing information needing to be updated and no US omission to trigger prediction error. Other studies have demonstrated disruption of reconsolidation without prediction error (e.g., [43, 44]), and there is variability in the boundary conditions proposed for reconsolidation to occur $[45,46]$. Our findings suggest that the precise 
conditions during retrieval may determine not only whether memories become labile but also which components of memory become susceptible to disruption.

Implications for conceptualizing the structure of Pavlovian memories

How might propranolol degrade the emotional/motivational component of a Pavlovian memory while leaving its ability to evoke other CRs intact? One possibility is that a CS may acquire separate associations with many different features of an US, including sensory, hedonic, motivational, temporal, and response features (e.g., see Fig. 1 in [47]). STs may develop a stronger leverCS-US motive association than GTs, and such an association may be weaker when a tone serves as the CS. Thus, if post-retrieval propranolol selectively disrupted a CS-US motive association, while leaving associations with other features of the US intact, this could explain the pattern of results reported here. However, why propranolol should disrupt the association between the CS and only one feature of the US is not clear.

Another possibility is that the incentive motivational value of a CS is not just an emergent property of a CS-US association, or due to a specific CS-US motive association, but requires a separate, modulatory process that values and revalues the CS dynamically as physiological state changes, even in the absence of new learning [48-51]. For example, one computational model includes incentive salience as a gain factor, ' $K$ ' [51], which is independent from the associative value of a CS. In another model changes in $\omega$, a variable that weighs which of two learning systems dominate control of behavior, can alter the tendency to sign-track or goaltrack, presumably in part by modulating incentive value [49]. None of these models perfectly capture the instantaneous changes in Pavlovian motivational value attributed to a CS as physiological state changes $[48,50]$, but such formulations raise the possibility that the effect of post-retrieval propranolol involves modulation of a non-associative "gain" factor that confers emotional/motivational value to CSs. At this point it is not clear which account explains the specificity of the effect of propranolol on some CRs and not on others, but the present results certainly challenge the notion that post-retrieval propranolol simply erases memories (e.g., see [34]).

Implications for the treatment of memory-related psychopathology

Propranolol has been used as a treatment to relieve pathological effects post-traumatic stress disorder [52, 53], as well as cravings elicited by stimuli in drug and alcohol addiction [54, 55]. However, the idea of "erasing memories" as a psychiatric treatment has spurred ethical concerns; one might imagine this treatment turning into a scenario in which people make the decision to erase memories to expunge their past ([56]; but see [57]). If disrupting reconsolidation decreases the emotional/motivational impact of a memory without erasure, this may both mitigate some of these ethical concerns and increase its therapeutic potential for psychiatric disorders characterized by maladaptive memories.

\section{ACKNOWLEDGEMENTS}

We thank Alex Kawa, Elizabeth O'Donnell, and Meghan Zechmeister for their assistance with behavioral testing, and Kyle Pitchers and Joshua Haight for assistance with the immunohistochemistry. Research supported by grant P01 DA031656 to TER. Authors report no financial interests or potential conflicts of interest.

\section{ADDITIONAL INFORMATION}

Supplementary information accompanies this paper at (https://doi.org/10.1038/ s41386-018-0082-0).

Competing interest: This work was supported by the National Institutes of Health
Grant P01 DA031656 to TER. All authors declare no financial and non-financial competing interests. The authors declare no conflict of interest.

Publisher's note: Springer Nature remains neutral with regard to jurisdictional claims in published maps and institutional affiliations.

\section{REFERENCES}

1. Nader K, Schafe GE, Le Doux JE. Fear memories require protein synthesis in the amygdala for reconsolidation after retrieval. Nature. 2000;406:722.

2. Tronson NC, Wiseman SL, Olausson P, Taylor JR. Bidirectional behavioral plasticity of memory reconsolidation depends on amygdalar protein kinase A. Nat Neurosci. 2006;9:167.

3. Alberini CM, LeDoux JE. Memory reconsolidation. Curr Biol. 2013;23:R746-50.

4. Milton AL, Everitt BJ. The psychological and neurochemical mechanisms of drug memory reconsolidation: implications for the treatment of addiction. Eur J Neurosci. 2010;31:2308-19.

5. Tronson NC, Taylor JR. Addiction: a drug-induced disorder of memory reconsolidation. Curr Opin Neurobiol. 2013;23:573-80.

6. Lee JL. Reconsolidation: maintaining memory relevance. Trends Neurosci. 2009;32:413-20.

7. Tronson NC, Taylor JR. Molecular mechanisms of memory reconsolidation. Nat Rev Neurosci. 2007:8:262-75.

8. Misanin JR, Miller RR, Lewis DJ. Retrograde amnesia produced by electroconvulsive shock after reactivation of a consolidated memory trace. Science. 1968;160:554-5.

9. Przybyslawski J, Roullet $\mathrm{P}$, Sara SJ. Attenuation of emotional and nonemotional memories after their reactivation: Role of $\beta$ adrenergic receptors. J Neurosci. 1999;19:6623-8.

10. Kindt $M$, Soeter $M$, Vervliet B. Beyond extinction: erasing human fear responses and preventing the return of fear. Nat Neurosci. 2009;12:256.

11. Soeter $M$, Kindt $M$. Dissociating response systems: erasing fear from memory. Neurobiol Learn Mem. 2010;94:30-41.

12. Bindra D. A unified interpretation of emotion and motivation. Ann N Y Acad Sci. 1969;159:1071-83.

13. Flagel SB, Robinson TE. Neurobiological basis of individual variation in stimulusreward learning. Curr Opin Behav Sci. 2017;13:178-85.

14. Robinson TE, Yager LM, Cogan ES, Saunders BT. On the motivational properties of reward cues: individual differences. Neuropharmacology. 2014;76:450-9.

15. Hearst $E$, Jenkins HM. Sign-tracking: the stimulus-reinforcer relation and directed action. Austin: Monograph of the Psychonomic Society; 1974.

16. Boakes RA. Performance on learning to associate a stimulus with positive reinforcement. In: Davis H, Hurwitz, H, editors. Operant-Pavlovian interactions. John Wiley \& Sons Inc.; Hillsdale, N.J. 1977. p. 67-97.

17. Robinson TE, Flagel SB. Dissociating the predictive and incentive motivational properties of reward-related cues through the study of individual differences. Biol Psychiatry. 2009;65:869-73.

18. Saunders BT, Robinson TE. A cocaine cue acts as an incentive stimulus in some but not others: implications for addiction. Biol Psychiatry. 2010;67:730-6.

19. Yager LM, Robinson TE. Cue-induced reinstatement of food seeking in rats that differ in their propensity to attribute incentive salience to food cues. Behav Brain Res. 2010;214:30-4.

20. Ahrens AM, Meyer PJ, Ferguson LM, Robinson TE, Aldridge JW. Neural activity in the ventral pallidum encodes variation in the incentive value of a reward cue. J Neurosci. 2016;36:7957-70.

21. Flagel SB, Cameron CM, Pickup KN, Watson SJ, Akil H, Robinson TE. A food predictive cue must be attributed with incentive salience for it to induce c-fos mRNA expression in cortico-striatal-thalamic brain regions. Neuroscience. 2011;196:80-96.

22. Yager LM, Pitchers KK, Flagel SB, Robinson TE. Individual variation in the motivational and neurobiological effects of an opioid cue. Neuropsychopharmacology. 2015;40:1269-77.

23. Olshavsky ME, Song B, Powell DJ, Jones CE, Monfils MH, Lee HJ. Updating appetitive memory during reconsolidation window: critical role of cue-directed behavior and amygdala central nucleus. Front Behav Neurosci. 2013;7:186.

24. Shumake J, Furgeson-Moreira S, Monfils MH. Predictability and heritability of individual differences in fear learning. Anim Cogn. 2014;17:1207-21.

25. Meyer PJ, Lovic V, Saunders BT, Yager LM, Flagel SB, Morrow JD, Robinson TE. Quantifying individual variation in the propensity to attribute incentive salience to reward cues. PLoS ONE. 2012;7:e38987.

26. Saunders BT, Robinson TE. The role of dopamine in the accumbens core in the expression of Pavlovian conditioned responses. Eur J Neurosci. 2012;36:2521-32.

27. Cleland GG, Davey GC. Autoshaping in the rat: the effects of localizable visual and auditory signals for food. J Exp Anal Behav. 1983;40:47-56. 
28. Meyer PJ, Cogan ES, Robinson TE. The form of a conditioned stimulus can influence the degree to which it acquires incentive motivational properties. PLoS ONE. 2014;9:e98163.

29. Beckmann JS, Chow JJ. Isolating the incentive salience of reward-associated stimuli: value, choice, and persistence. Learn Mem. 2015;22:116-27.

30. Singer BF, Bryan MA, Popov P, Scarff R, Carter C, Wright E, Aragona BJ, Robinson TE. The sensory features of a food cue influence its ability to act as an incentive stimulus and evoke dopamine release in the nucleus accumbens core. Learn Mem. 2016;23:595-606.

31. Milton AL, Lee JL, Everitt BJ. Reconsolidation of appetitive memories for both natural and drug reinforcement is dependent on $\beta$-adrenergic receptors. Learn Mem. 2008;15:88-92.

32. Robinson $\mathrm{M}$, Franklin $\mathrm{K}$. Central but not peripheral beta-adrenergic antagonism blocks reconsolidation for a morphine place preference. Behav Brain Res. 2007;182:129-34.

33. Vetere G, Piserchia V, Borreca A, Novembre G, Aceti M, Ammassari-Teule M. Reactivating fear memory under propranolol resets pre-trauma levels of dendritic spines in basolateral amygdala but not dorsal hippocampus neurons. Front Behav Neurosci. 2013;7:211

34. Sandkühler J, Lee J. How to erase memory traces of pain and fear. Trends Neurosci. 2013;36:343-52.

35. Bouton ME, Moody EW. Memory processes in classical conditioning. Neurosci Biobehav Rev. 2004;28:663-74.

36. Villain H, Benkahoul A, Drougard A, Lafragette M, Muzotte E, Pech S, Bui E, Brunet $A$, Birmes $P$, Roullet $P$. Effects of propranolol, a $\beta$-noradrenergic antagonist, on memory consolidation and reconsolidation in mice. Front Behav Neurosci. 2016;10:49

37. Cardinal RN, Parkinson JA, Hall J, Everitt BJ. Emotion and motivation: the role of the amygdala, ventral striatum, and prefrontal cortex. Neurosci Biobehav Rev. 2002;26:321-52.

38. Flagel SB, Clark JJ, Robinson TE, Mayo L, Czuj A, Willuhn I, Akers CA, Clinton SM, Phillips $\mathrm{PE}$, Akil H. A selective role for dopamine in stimulus-reward learning. Nature. 2011;469:53-7.

39. Blaiss CA, Janak PH. Post-training, but not post-reactivation, administration of amphetamine and anisomycin modulates Pavlovian conditioned approach. Neurobiol Learn Mem. 2007;87:644-58.

40. Reichelt AC, Lee JL. Memory reconsolidation in aversive and appetitive settings. Front Behav Neurosci. 2013;7:118

41. Pasquariello KZ, Han M, Unal C, Meyer PJ. Adrenergic manipulation inhibits Pavlovian conditioned approach behaviors. Behav Brain Res. 2018;339:278-85.

42. Fernández RS, Boccia MM, Pedreira ME. The fate of memory: reconsolidation and the case of prediction error. Neurosci Biobehav Rev. 2016;68:423-41.
43. Duvarci S, Nader K. Characterization of fear memory reconsolidation. J Neurosci. 2004;24:9269-75.

44. Dunbar AB, Taylor JR. Garcinol blocks the reconsolidation of multiple cocainepaired cues after a single cocaine-reactivation session. Neuropsychopharmacology. 2017;42:1884.

45. Finnie PS, Nader K. The role of metaplasticity mechanisms in regulating memory destabilization and reconsolidation. Neurosci Biobehav Rev. 2012;36:1667-707.

46. Elsey JW, Kindt M. Tackling maladaptive memories through reconsolidation: from neural to clinical science. Neurobiol Learn Mem. 2017;142:108-17.

47. Delamater AR, Oakeshott $S$. Learning about multiple attributes of reward in Pavlovian conditioning. Ann N Y Acad Sci. 2007;1104:1-20.

48. Dayan $P$, Berridge KC. Model-based and model-free Pavlovian reward learning: revaluation, revision, and revelation. Cogn Affect Behav Neurosci. 2014;14:473-92.

49. Lesaint $F$, Sigaud $O$, Flagel SB, Robinson TE, Khamassi M. Modelling individual differences in the form of pavlovian conditioned approach responses: a dual learning systems approach with factored representations. PLoS Comput Biol. 2014;10:e1003466.

50. Robinson MJF, Berridge KC. Instant transformation of learned repulsion into motivational "wanting". Curr Biol. 2013;23:282-9.

51. Zhang J, Berridge KC, Tindell AJ, Smith KS, Aldridge JW. A neural computational model of incentive salience. PLoS Comput Biol. 2009;5:e1000437.

52. Mahabir M, Tucholka A, Shin LM, Etienne P, Brunet A. Emotional face processing in post-traumatic stress disorder after reconsolidation impairment using propranolol: a pilot fMRI study. J Anxiety Disord. 2015;36:127-33.

53. Brunet A, Poundja J, Tremblay J, Bui É, Thomas É, Orr SP, Azzoug A, Birmes P, Pitman RK. Trauma reactivation under the influence of propranolol decreases posttraumatic stress symptoms and disorder: 3 open-label trials. J Clin Psychopharmacol. 2011;31:547-50.

54. Lonergan MH, Saumier D, Tremblay J, Kieffer B, Brown TG, Brunet A. Reactivating addiction-related memories under propranolol to reduce craving: a pilot randomized controlled trial. J Behav Ther Exp Psychiatry. 2016;50:245-9.

55. Saladin ME, Gray K, Abbott T, LaRowe S, McRae-Clark A, DeSantis S, Baker N, Back S, Hartwell K, Brady KT. Post-retrieval propranolol may alter reconsolidation of trauma memory in individuals with PTSD and comorbid alcohol dependence. Drug Alcohol Depend. 2014;140:e193.

56. Kolber AJ. Therapeutic forgetting: the legal and ethical implications of memory dampening. Vand L Rev. 2006;59:1559.

57. Kolber A. Neuroethics: give memory-altering drugs a chance. Nature 2011;476:275-6. 